Support statement: The Wheezing Illnesses STudy LEidsche Rijn received grant 2100.0095 from The Netherlands Organisation for Health Research and Development (ZonMw). An unrestricted grant was received from GlaxoSmithKline. Additionally, this work was financially supported by the UMC Utrecht Vascular Prevention Project.

Conflict of interest: Disclosures can be found alongside the online version of this article at www.erj.ersjournals.com

Acknowledgements: The authors gratefully acknowledge all parents and children who participated, C. Geerts (Julius Center for Health Sciences and Primary Care, University Medical Center Utrecht, Utrecht, the Netherlands) and L. van der Feltz-Minkema (Dept of Paediatric Pulmonology, Wilhelmina Children's Hospital, University Medical Center Utrecht, Utrecht) for their major contribution to the data collection, M. Olling-de Kok (Dept of Paediatric Pulmonology, Wilhelmina Children's Hospital, University Medical Center Utrecht, Utrecht) for secretarial support, J. Zwerver (Julius Center for Health Sciences and Primary Care, University Medical Center Utrecht, Utrecht) for data management and the Vascular Imaging Centre for assistance in the vascular measurements.

\title{
References
}

1 Bruno A, Pace E, Chanez P, et al. Leptin and leptin receptor expression in asthma. J Allergy Clin Immunol 2009; 124 : 230-237.

2 Bergen HT, Cherlet TC, Manuel P, et al. Identification of leptin receptors in lung and isolated fetal type II cells. Am J Respir Cell Mol Biol 2002; 27: 71-77.

3 Nair P, Radford K, Fanat A, et al. The effects of leptin on airway smooth muscle responses. Am J Respir Cell Mol Biol 2008; 39: 475-481.

4 Katier N, Uiterwaal CSPM, de Jong BM, et al. The Wheezing Illnesses Study Leidsche Rijn (WHISTLER): rationale and design. Eur J Epidemiol 2004; 19: 895-903.

5 Evelein AMV, Visseren FLJ, van der Ent CK, et al. Excess early postnatal weight gain leads to increased abdominal fat in young children. Int J Pediatr 2012; 2012: 141656.

6 Schipper HS, de Jager W, van Dijk ME, et al. A multiplex immunoassay for human adipokine profiling. Clin Chem 2010; 56: 1320-1328.

7 Huang K, Rabold R, Abston E, et al. Effects of leptin deficiency on postnatal lung development in mice. J Appl Physiol (1985) 2008; 105: 249-259.

8 Arteaga-Solis E, Zee T, Emala CW, et al. Inhibition of leptin regulation of parasympathetic signaling as a cause of extreme body weight-associated asthma. Cell Metab 2013; 17: 35-48.

9 Kim KW, Shin YH, Lee KE, et al. Relationship between adipokines and manifestations of childhood asthma. Pediatr Allergy Immunol 2008; 19: 535-540.

10 Sin DD, Man SFP. Impaired lung function and serum leptin in men and women with normal body weight: a population based study. Thorax 2003; 58: 695-698.

11 Naveed B, Weiden MD, Kwon S, et al. Metabolic syndrome biomarkers predict lung function impairment: a nested case-control study. Am J Respir Crit Care Med, 185: 392-399.

12 Narayanan M, Owers-Bradley J, Beardsmore CS, et al. Alveolarization continues during childhood and adolescence: new evidence from helium-3 magnetic resonance. Am J Respir Crit Care Med 2012; 185: 186-191.

\section{The association between public transport and active tuberculosis in Lima, Peru}

To the Editor:

While there have been impressive gains in the global control of tuberculosis (TB) over the past two decades, TB remains a leading cause of death and efforts to decrease its burden have been limited by the rise of drug resistant strains [1]. As drug-resistant TB remains exceedingly difficult and costly to treat, more research is needed to identify areas for improving primary prevention of TB.

The risk of TB transmission is increased whenever there is overcrowding, poor ventilation and exposure to an infected individual, and public transport has been identified as a potential setting with increased risk for TB transmission [2]. Indeed, recent research demonstrates the fraction of rebreathed air on public transport is mathematically correlated with a higher risk of contracting TB [3].

Previous investigations using cross-sectional data in Lima, Peru, have demonstrated that community, rather than household, transmission may account for up to $70 \%$ of incident infections [4]. Studies conducted in Lima found an increased risk of TB infection among individuals who rode minibuses [5] and those who worked on public transport [6]. However, these studies were limited by misclassification of TB diagnosis, imprecise time variables and wide confidence intervals. 
The objective of our study was to assess the association between use of public transportation and active TB using a detailed transportation questionnaire, multiple control groups and improved TB diagnostics.

We used a matched case-control design and enrolled treatment-naïve individuals newly diagnosed with TB on the day of diagnosis from three peripheral health centres in the Lima metropolitan area. Three controls without TB were individually matched by age and sex for each incident case: a patient with a symptomatic respiratory syndrome from the same clinic as the incident case, a person living in the same household as the case and a person from the same neighbourhood as the case using random number sampling. Controls were excluded if they had a prior history of TB, were HIV positive or pregnant, or were currently living with anyone with active TB (with the exception the incident case for household controls). Cases and symptomatic controls underwent confirmatory testing with solid and liquid cultures to decrease misclassification.

Each participant completed a survey assessing demographic and socioeconomic factors, risk factors for TB and transport habits. As transport usage was likely to vary throughout the year, subjects were also asked about transport usage during the prior week. To reduce confounding by indication, cases and symptomatic respiratory controls were asked about transport usage the week before onset of respiratory symptoms. The association between use of public transport and active TB was modelled with logistic regression. Each covariate was evaluated and included in the model if it had an independent association between cases and controls $(p \leqslant 0.05)$ and all covariates were tested for multicollinearity. Time spent on public transport was converted to quintiles due to skewing of the mean by public transport workers, who reported spending $>920$ min per week on public transport.

We enrolled 86 cases from 86 households situated in 86 neighbourhoods and 85 symptomatic respiratory syndrome controls. Control groups did not vary significantly from each other and were therefore pooled in the analysis. Compared with controls, cases had lower body mass index (BMI), had a history of exposure to a household member with TB, differing occupations and more frequent travel between 15:00 $\mathrm{h}$ and 18:59 h. Other TB risk factors including income, number of household inhabitants, years of education, bacille Calmette-Guérin history, alcohol or tobacco use, diabetes and perception of TB exposure outside of the home did not significantly vary between case and control. There was also no significant difference between transport types. The significant covariates were included in a multivariate logistic regression model to assess transportation during the past week or past year with the risk of developing TB (table 1).

In the model assessing transport usage during the prior week, an increase in one quintile of time spent in public transportation increased the odds ratio of having TB by $34 \%$ (OR 1.34, 95\% CI 1.08-1.65; $\mathrm{p}=0.007$ ). There was also an association between transport usage over the past year and risk of TB, but it was not significant (OR 1.19, 95\% CI 0.97-1.46; $\mathrm{p}=0.093$ ). Lower BMI, previous cohabitation with a person with $\mathrm{TB}$ and being divorced or widowed were associated with a higher risk of TB, while working as a professional in professions requiring certification, such as healthcare, business and education, was associated with a lower risk of TB. There was an association between increased risk of TB with travel during 15:00 $\mathrm{h}$ and 18:59 h, which was statistically significant for the year-long model, but not for the past-week model. There was no significant effect modification among any of the covariates tested with duration of time spent on public transport.

Our study design decreased misclassification of active TB by using highly sensitive and specific TB culturing techniques, recruiting a larger sample size and using a detailed survey of public transport parameters. These attributes and the control of the known TB risk factors of prior cohabitation with a person infected with TB and BMI [7] lend further support to the likelihood that public transport is an independent risk factor for TB transmission. While risk of TB from prior household contact exceeded the risk associated with transport usage, our study did not assess other potential venues of community TB transmission, such as workplace environments, sporting events and marketplaces.

Although our analysis did not detect a significant association between travel duration and TB over the yearlong period, this information was self-reported and, therefore, resulted in a simplified estimation of transport usage over the past year, leading to nondifferential misclassification of the exposure towards the null. This is a documented challenge among questionnaires that require recall over a year-long period [8]. A more accurate estimation of transport usage would require the use of prospective travel logs, but this was not feasible for the scope of this study.

In the year-long mode, there was an increased risk of $\mathrm{TB}$ among those travelling between the hours of 15:00 $\mathrm{h}$ and 18:59 $\mathrm{h}$. This time frame encompasses the typical evening rush hour period in Lima, where there is increased transport usage and overcrowding. This finding suggests that certain routes and time frames may be linked with increased TB risk and serve as a medium for transmitting TB to other neighbourhoods. This hypothesis is supported by the finding in another study that specific bus routes traverse areas clustered with TB [9]. 
TABLE 1 Adjusted association between time spent on public transport and active pulmonary tuberculosis (TB) in Lima, Peru

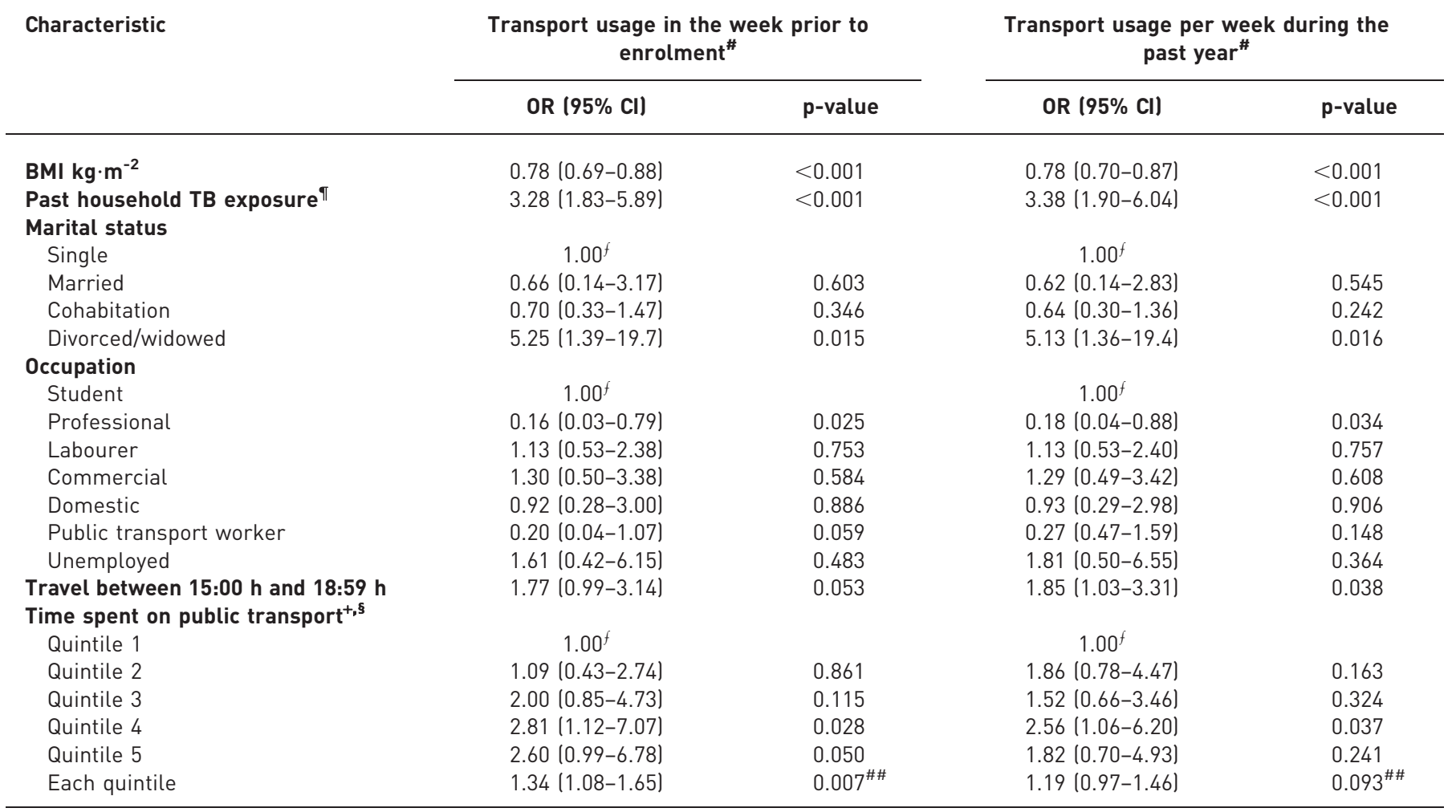

BMI: body mass index. ${ }^{*}: \mathrm{n}=343 .{ }^{\circ}$ : living with someone who had TB previously. ${ }^{+}$: during past week: quintile 1, $<120$ min; quintile 2, $120-279$ min; quintile 3, 280-539 $\mathrm{min}$; quintile 4, 540-919 $\mathrm{min}$; and quintile $5, \geqslant 920 \mathrm{~min} .{ }^{\S}$ : per week during the past year: quintile 1, <180 min; quintile 2, $180-$ $319 \mathrm{~min}$; quintile $3,320-599 \mathrm{~min}$; quintile 4,600-899 $\mathrm{min}$; and quintile $5 \geqslant 900 \mathrm{~min} .{ }^{f}$ : reference. ${ }^{\# \#}$ : for trend.

With respect to the post-2015 World Health Organization goal of TB elimination, defined as fewer than one case per million population, by 2050, identifying and prioritising treatment of individuals with latent $\mathrm{TB}$ at high risk of progression is essential to prevention [10]. Given the demonstrated risk conveyed by prior household TB exposure, our study affirms the importance of household contact tracing for TB and latent TB case finding and treatment. It also identifies public transportation as a source of community transmission. Further study of the correlation of transport time, type, route and degree of congestion with the risk of $\mathrm{TB}$ is merited to identify targeted preventative interventions and ascertain the role of these interventions in existing prevention programmes.

@ERSpublications

Case control study in Lima, Peru correlates public transport usage with developing active TB http://ow.ly/swlut

Nathan Woo Furukawa ${ }^{1}$, Alberto Mendoza-Ticona ${ }^{2}$, Jorge O. Alarcón-Villaverde ${ }^{3}$, Harrison Montejo ${ }^{2}$, Mark A. Micek ${ }^{1,4}$ and Joseph R. Zunt ${ }^{1}$

${ }^{1}$ Dept of Global Health, University of Washington, Seattle, WA, and ${ }^{4}$ Dept of Medicine, University of WisconsinMadison, Madison, WI, USA. ${ }^{2}$ Instituto Nacional de Salud, Centro Nacional de Salud Pública, Lima, and ${ }^{3}$ Universidad Nacional Mayor de San Marcos, Instituto de Medicina Tropical Daniel A. Carrión, Lima, Peru.

Correspondence: N.W. Furukawa, 1905 E Fir St, Seattle, WA 98122, USA. E-mail: furukn1@uw.edu

Received: Sept 192013 | Accepted after revision: Nov 172013 | First published online: Dec 52013

Support statement: This study was funded and supported by the University of Washington Dept of Global Health, Instituto Nacional de Salud of Peru, and University of San Marcos.

Conflict of interest: None declared.

Acknowledgements: The authors thank Lizbeth Hidalgo and Juan Carlos Llontop (Dirección de Salud IV Lima Este, Lima, Peru); Elizabeth Peinado, Elena Castillo and Amelia Chavez (Dirección de Salud II Lima Sur, Lima); Osvaldo Mariñas 
(Instituto Nacional de Salud, Centro Nacional de Salud Pública, Lima); Edith Castillo (Direccion Regional de Salud del Callao, Laboratorio de Referencia Regional de Tuberculosis, Callao, Peru); and Thanh Ton (Dept of Global Health, University of Washington, Seattle, WA, USA) for their contribution to this work.

\title{
References
}

World Health Organization. Global tuberculosis report 2013. www.who.int/tb/publications/global_report/en/ Date last accessed: January 8, 2014.

2 Edelson PJ, Phypers M. TB transmission on public transportation: a review of published studies and recommendations for contact tracing. Travel Med Infect Dis 2011; 9: 27-31.

3 Andrews JR, Morrow C, Wood R. Modeling the role of public transport in sustaining tuberculosis transmission in South Africa. Am J Epidemiol 2013; 177: 556-561.

4 Brooks-Pollock E, Becerra MC, Goldstein E, et al. Epidemiologic inference from the distribution of tuberculosis cases in households in Lima, Peru. J Infect Dis 2011; 203: 1582-1589.

5 Horna-Campos OJ, Sanchez-Perez HJ, Sanchez I, et al. Public Transportation and Pulmonary Tuberculosis, Lima, Peru. Emerg Infect Dis 2007; 13: 1491-1493.

6 Horna-Campos OJ, Consiglio E, Sanchez-Perez HJ, et al. Pulmonary tuberculosis infection among workers in the informal public transport sector in Lima, Peru. Occup Environ Med 2010; 68: 163-165.

7 Brewer TF, Choi HW, Seas C, et al. Self-reported risks for multiple-drug resistance among new tuberculosis cases: Implications for drug susceptibility screening and treatment. PLoS One 2011; 6: e25861.

8 Joachim G. Sources of variability in the reproducibility of food frequency questionnaires. Nutr Health 1998; 12: 181-188.

9 Feske ML, Teeter LD, Musser JM, et al. Giving TB wheels: Public transportation as a risk factor for tuberculosis transmission. Tuberculosis (Edinb) 2011; 91: Suppl. 1, S16-S23.

10 Diel R, Loddenkemper R, Zellweger J, et al. Old ideas to innovate tuberculosis control: preventive treatment to achieve elimination. Eur Respir J 2013; 42: 785-801.

\section{Diffusion capacity and BMPR2 mutations in pulmonary arterial hypertension}

\author{
To the Editor:
}

Pulmonary arterial hypertension (PAH) is a disease in which remodelling of the small pulmonary arteries leads to an increase in pulmonary artery pressure (PAP). The most important genetic predisposing factor related to $\mathrm{PAH}$ is a mutation in the bone morphogenetic protein receptor type 2 gene $(B M P R 2)[1,2]$. BMPR2 mutation carriers are known to present with disease at an earlier age and with worse haemodynamics [3]. We recently showed in a cohort of patients with idiopathic and hereditary PAH that a very low diffusion capacity for carbon monoxide (DLCO) is exclusively found in some of the patients without identified BMPR2 mutations, whereas BMPR2 mutation carriers have a relatively preserved DLCO [4]. DLCO is a noninvasive marker of the quality of the alveolar capillary structure [5] and the observed difference in DLCO supports the hypothesis that distinct vascular disease processes are at play in BMPR2 mutation-related PAH and non BMPR2 mutation-related idiopathic PAH. Until recently, insufficient availability of lung samples has prohibited the performance of a detailed comparison of the pulmonary vascular pathologies in these two disease groups [6]. Therefore, we sought, in the present study, to confirm the previously found influence of $B M P R 2$ mutations on diffusion capacity in a much larger multinational patient cohort.

We performed a retrospective collaborative study at the VU University Medical Center in Amsterdam, the Netherlands and the Université Paris-Sud, Assistance Publique Hôpitaux de Paris, Le Kremlin-Bicêtre, France. Patients were eligible for this study when classified in the database with idiopathic or familial PAH, and when the results from BMPR2 mutation analysis and DLCO measurements were available. Patients were diagnosed with idiopathic PAH according to current clinical guidelines [7]. Familial PAH was diagnosed when at least one family member had confirmed PAH. Patients with a family history of PAH and no mutations identified in the BMPR2 gene were not included in this study. In total 64 patients were selected from the Dutch idiopathic and familial PAH population and 85 patients were drawn from the French population. Comorbidities of all these patients were reviewed, as was the amount of tobacco exposure. In addition, patients were reassessed for the likelihood of pulmonary veno-occlusive disease (PVOD). Patients were excluded when they had a tobacco exposure $>20$ pack-years or a medical history mentioning

For editorial comments see page 963. 